\title{
Verhaltensdispositionen I: Die biologische Perspektive
}

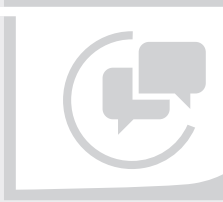

festationen sind am ehesten als Wirkungen ähnlicher Ursachen anzusehen. Dafür gibt es viele Beispiele - von den Reflexen, über den Einfluss diverser Hormonspiegel bis zur Steuerung der Schlafstadienwechsel. Zum Dritten schließlich haben Mechanismen den Vorteil, die Latenz von Verhaltensdispositionen zu erklären: Die jeweiligen Mechanismen „produzieren“ ihre Wirkungen nur, wenn sie zum Einsatz kommen. Wenn Mechanismen hingegen „stumm“ bleiben, dann entspricht dies der Latenz einer sich nicht manifestierenden Disposition.

Allem Anschein nach verfährt unsere intuitive Dispositions-Psychologie analog zur ausgeführten Dispositions-Physiologie: Wir verstehen psychologische Eigenschaften bzw. kategorisierbares Verhalten generell (!) als Ausdruck von Verhaltensdispositionen. Denn auch die Psyche wirkt mittels interner Verhaltensdispositionen von „innen nach außen“. Die unterschiedenen Persönlichkeitseigenschaften, Appetenzen, Reaktionsneigungen etc. „bewegen“ die Person zu einem bestimmten Verhalten. Damit folgt unsere Dispositions-Psychologie der Alltagspsychologie, die Verhalten als Handeln aufgrund innerer Bedingungen unter gegebenen Umständen begreift. Da sowohl die inneren als auch die äußeren Bedingungen ständig variieren, ist es naheliegend, trans-situational „stabiles“ Verhalten auf stabile Verhaltensdispositionen zurückzuführen.

Betrachten wir ein Beispiel: Warum hatte Hans gestern Abend einen Wutanfall? Nun, Hans gilt als temperamentvolle Person. Er war mit Lisa aneinandergeraten, die ihm, wie er meinte, ungerechtfertigte Vorwürfe machte. Trotz aufsteigenden Ärgers hatte er hin und her überlegt, wie er die Situation am besten bewältigen könne. Schließlich ist es ihm „zu bunt“ geworden - will sagen: Hans wurde von Wut „überwältigt“.

Alle psychologischen Eigenschaften in solchen Beispielen sind dispositionaler Art. Die mentalen Zustände, psychischen Struk- turen und Prozesse, auf die wir erklärend verweisen, sind latente Verhaltensbereitschaften, die sich aus bestimmten Gründen manifestiert haben - dies aber nicht tun müssen: Man kann eine temperamentvolle Persönlichkeit sein und sich trotzdem bei Gelegenheit zurückhalten. Eine Person kann eine Situation je nach Umständen unterschiedlich erleben, auffassen und begreifen. Ein Gleiches gilt für die Denkfähigkeit einer Person, die ein breites Spektrum an Denkprozessen generieren kann. Und auch die Gefühlsreaktionen und deren Regulation variieren in Abhängigkeit von gegebenen Bedingungen. Es lohnt die Übung, einmal nach „nicht dispositionalen“- die Philosophen sagen: „kategorialen“ - psychologischen Attributen zu suchen: Gibt es psychologische Eigenschaften, die analog zu kategorialen Eigenschaften wie „Körpergröße“, „rot“ oder „dreieckig“ nicht situativ variieren, weil sie nicht davon abhängen, wie eine Person gerade „tickt“?

Ich werde im Folgenden einen kritischen Blick auf den Versuch werfen, die Physiologie menschlichen Verhaltens anhand von transsituational wirksamen Verhaltensdispositionen aufzuklären. Denn was im Falle robuster Organdispositionen etwa des Dickdarms oder der Schilddrüse gut funktioniert, erweist sich im Falle des Gehirns und seiner flexiblen Aktivität als extrem schwierig, was neben der Komplexität der Gehirnaktivität womöglich mit den folgenden theoretischen Problemen zusammenhängt:

- Verhaltensdispositionen sind hypothetische Größen, die sich nicht direkt beobachten lassen. Sie lassen sich nicht anhand des Verhaltens „von außen“ beobachten, weil das Verhalten nur Merkmale der Manifestation, nicht aber Merkmale der Disposition selber aufweist. Und sie lassen sich nicht ohne Weiteres „von innen“ anhand der Aktivität physiologischer Prozesse beobachten, weil die Aktualisierung ihrer kausalen Rolle von den Umständen ab- 
hängt. Daraus folgt, dass Dispositionszuschreibungen im besonderen Maße theorie- bzw. interpretationsabhängig erfolgen.

- Der hypothetische Charakter der Verhaltensdispositionen offenbart ein Dilemma: Einerseits sind Verhaltensdispositionen unumgänglich. Wir nutzen nun einmal Dispositionshypothesen, um Verhalten als Handeln zu verstehen und zu analysieren. Andererseits sind Dispositionshypothesen „zu billig“ zu haben: Die Art, wie wir Verhaltensdispositionen individuieren, lädt zu Willkür und Beliebigkeit ein. Dies liegt nicht zuletzt an der Tendenz, die Manifestationen einer (mutmaßlichen) Disposition als Beleg für die Erfüllung der kausalen Rolle der Disposition zu nehmen - wie es Molières Doktor vorgeführt hat.

\section{Die biomedizinische Perspektive}

Die moderne Physiologie betrachtet den Organismus als ein kausales System, dass sich aus einer Vielzahl von Mechanismen zusammensetzt. Um dieses „komplexe Uhrwerk" zu entschlüsseln, muss man zunächst ermitteln, was der Organismus tut bzw. tun kann. Dies erfolgt in der Form von Dispositionshypothesen, die die Suche nach den implementierenden Mechanismen leiten. Denn wenn man nicht weiß, WAS es aufzuklären gilt, können Bemühungen um das WIE und WARUM nicht greifen. Im Wechselspiel endloser Variationen aus Dispositionszuschreibung und experimenteller Intervention im Dienste einer „rekursiven mechanistischen Analyse“ ist es in vielen Fällen gelungen, die Mechanismen des Organismus zu lokalisieren, zu dekomponieren und ihre kausalen Regularitäten aufzuklären. Doch auch wenn man die Mechanismen im Grundsatz kennt, bleibt es schwierig, die konkreten Operationen dieser Mechanismen im situativen Kontext zu erfassen. Wie greifen, um das Beispiel zu bemühen, die Aktivitäten des autonomen Nervensystems und der Stress-, Hormonund Emotions-(Regulations-)systeme im Fall von Hans' Wutanfall ineinander? Da sich physiologische Mechanismen nur in „dekontextualisierter“ Form „sauber“ messen lassen, wirft eine ökologisch validere Analyse enorme methodische Probleme auf.
Im Fokus psychiatrischer Forschung stehen die psychischen Störungen: Man versucht zunächst, Regularitäten im vieldimensionalen Flux klinischer Phänomene zu erkennen, zu beschreiben und zu kategorisieren. Das Ergebnis sind die etablierten nosologischen Taxa wie etwa Depression oder Schizophrenie. Dann werden Hypothesen über die Dispositionen formuliert, die für die Manifestation dieser phänomenalen Regularitäten verantwortlich sein könnten. Entsprechende Beispiele reichen von genetischen, neurotypischen oder dysontogenetischen Risiko-Dispositionen bis zu psychologischen Dispositionshypothesen wie „Neurotizismus“ oder „Schizotaxie“, die mit bestimmten Störungen kovariieren sollen. Idealerweise sollte es eines Tages gelingen, die Mechanismen, die die kausalen Rollen dieser pathogenen Dispositionen erfüllen, zu identifizieren, zu lokalisieren und schrittweise zu dekomponieren [1].

\section{Molières Doktor}

Die Attribution von Verhaltensdispositionen changiert kaum merklich zwischen 2 Ebenen. Die Rede von solchen Dispositionen bezieht sich mal mehr auf die Ebene der Verhaltensmanifestationen und mal mehr auf die Ebene mutmaßlicher psychophysiologischer Prozesse, die diesen Manifestationen zugrunde liegt. Wenn es etwa heißt, „Lisa ist extrovertiert!“, dann bedeutet dies gemäß der „Manifestations-Lesart“ so viel wie: Wenn Lisa in Gesellschaft ist, dann geht sie auf die Leute zu, redet viel, freundet sich schnell an, hat positive Gefühle etc. Lisas „Extrovertiertheit“ meint in diesem Fall die regelhafte Manifestation ihrer Persönlichkeit. Wenn man hingegen Extrovertiertheit als verhaltensbestimmenden „Trait“ versteht, dann bezieht sich die Aussage, „Lisa ist extrovertiert“, auf Lisas Persönlichkeitsdisposition unabhängig von deren Manifestationen. Lisa ist so gesehen auch dann extrovertiert, auch wenn sie sich gerade nicht extrovertiert verhält.

Diese, auf den ersten Blick harmlos erscheinende Doppeldeutigkeit ruft den berühmten Doktor aus Molières Stück „Der eingebildete Kranke“ auf den Plan: Auf die Frage, warum Opium die Patienten in Schlaf versetzt, antwortet der Doktor: Weil dem Opium der „virtus dormitiva“ innewohne. Die Einnahme von Opium, so seine „dispo- sitionale Erklärung“, lasse die Patienten einschlafen (Manifestation), weil dem Opium die „Tugend des Schlafenmachens“ eigen sei (Disposition). Voltaires ironische Absicht, das irrationale Denken der Ärzte seiner Zeit bloßzustellen, ist keineswegs erledigt: Denn auch wir neigen dazu, wenn wir uns auf die Ebene beobachtbarer phänomenaler Manifestationen beziehen, zugleich theoretische Annahmen über deren kausale Basis ins Spiel zu bringen. Doch die zirkuläre Reformulierung des Offensichtlichen - Opium macht schlafen, weil Opium die Eigenschaft hat, schlafen zu machen - beinhaltet keinen Erkenntnisgewinn. Die Reifikation der phänomenalen Regularität auf der Ursachen-Ebene ist vielmehr irreführend, weil sie ein Wissen suggeriert, dass nicht existiert. Es handelt sich bei vielen Dispositionshypothesen um pseudowissenschaftlichen Bluff, der alles andere als überwunden ist, wie folgende Beispiele andeuten mögen.

Erweist sich eine Person als übermäßig gewissenhaft, ja zwanghaft, so wird dafür (von manchen) ein „strenges Über-Ich“ verantwortlich gemacht. Doch der Rekurs auf die hypothetische Persönlichkeitsinstanz „Über-Ich“ reifiziert nur, was wir bereits wissen. Gewissenhaftes, ja zwanghaftes Verhalten mit dem „Laster des Zwangs“ - einem „vitium obsessum“ gewissermaßen - zu erklären, trägt nichts zum Verständnis der Gewissenhaftigkeit des Betroffenen bei. Ähnlich verhält es sich mit der verbreiteten Überzeugung, die Kernpathologie der Borderline-Persönlichkeitsstörung bestehe in einer „Emotionsregulationsstörung“. Wir diagnostizieren die genannte Persönlichkeitsstörung definitionsgemäß als „ein tiefgreifendes Muster von Instabilität in zwischenmenschlichen Beziehungen, im Selbstbild und in den Affekten " [2] und erklären das Auftreten dieses Syndroms als Ausdruck einer Disposition, die sich in instabilen zwischenmenschlichen Beziehungen, einem instabilen Selbstbild und stark wechselnden Affekten manifestiert. Da es, nebenbei bemerkt, kaum psychische Störungen gibt, die nicht durch Defizite der Emotionsregulation bzw. ein „vitium affectus mali“ gekennzeichnet sind, wird deutlich: Die Neigung, phänomenale Regularitäten als unterscheidbare, ontologisch robuste Dispositionen zu reifizieren, behindert den Erkenntnisfortschritt mehr, 
als dass sie diesen fördert. Wir müssten, um dem zu begegnen, anfangen, den eigenen Jargon „klinischer Eigentlichkeit“ kritisch zu hinterfragen. Dass wir von diesem Ziel noch weit entfernt sind, mag der geradezu ubiquitäre Verweis auf „Informationsverarbeitungsstörungen“ illustrieren. Wenn wir kognitive Prozesse als Informationsverarbeitungsprozesse definieren, dann sind alle, als „kognitive Störungen“ erkannten Psychopathologien automatisch Informationsverarbeitungsstörungen. Dies ist ungefähr so erhellend, wie die Feststellung, dass es sich bei einem halbvollen Glas Bier um ein informationsverarbeitendes System handelt, da dieses ja seinen Füllungsstand anzeige.

Dabei ist lange bekannt, wie das Problem „zirkulärer Reifikation“ zu lösen wäre: Wir sollten unsere Dispositionshypothesen nach Möglichkeit in einer zugrunde liegenden Basis empirisch „verankern“. Dadurch würde nicht nur der leichtfertige Gebrauch „notationeller Varianten“ eingeschränkt. Wir würden auch besser verstehen, dass theoretische Schlussfolgerungen, wie „Gewissenhaftigkeit ist Ausdruck des Über-Ich“ oder „Borderline-Symptomatik ist Folge von Emotionsregulationsstörungen“ nur zulässig sind, wenn wir „Über-Ich“ oder „Emotionsregulationsstörungen“ logisch unabhängig vom zu erklärenden Verhalten, anhand eigenständiger Evidenzklassen zuvor begründet haben.

Wie dies gelingen kann, illustriert die Pharmakodynamik des Opiums: Opium enthält u. a. Morphin, das an zentralnervösen Opioidrezeptoren agonistisch wirkt und seine psychotropen Wirkungen auf diesem Weg entfaltet. Im Falle des „Über-Ichs“ wäre grundsätzlich ein „Gewissens-Parameter“ denkbar - verkörpert durch ein Funktionsmodul der „universellen kognitiven Architektur des menschlichen Geistes “ -, dessen Existenz und Wirkungsweise anhand diverser unabhängiger Evidenzklassen nachzuweisen wäre. Im Falle der Emotionsregulation könnten es präfrontale Netzwerkaktivitäten sein, die die Aktivität sub-kortikaler Netzwerke modulieren. Würde sich zeigen, dass diese Netzwerke bei „Borderline-Patienten " weniger entwickelt sind, wäre dies sehr überzeugend.

Doch der Forschungstand: Er ist nicht so. Wir kennen weder die „kognitive Architek- tur des Geistes“. Noch herrscht Einigkeit darüber, wie eine „Karte“ derselben zu erstellen wäre. Und die Rede von spezifischen Gehirnnetzwerken und deren funktioneller Rolle krankt an der systematischen Konfundierung von experimenteller „Aufgabe“ und gemessener „Aktivität“: Die Tendenz von taskabhängigen Netzwerkaktivierungen auf eine speziestypische funktionelle Organisation des Nervensystems zu schließen, führt meist in die Irre. Denn valide Interpretationen gemessener Aktivitäten hätten zur Voraussetzung, dass man die Struktur-Funktions-Beziehungen des Gehirns bereits kennt, und die Befunde entsprechend einordnen kann [3]. Da diese Voraussetzung nicht gegeben ist, nimmt es nicht Wunder, dass die neuro-psychiatrische Forschung in vielen Bereichen auf der Stelle tritt, weil sich ein „Durchbruch“ nach dem anderen als falsch positiver Befund erweist oder sich nicht replizieren lässt.

\section{Biologischer Realismus}

Trotz der erwähnten Probleme ist die Attribution von Dispositionen eine Grundübung der Verhaltenswissenschaften. Die meisten Forscher vertreten einen robusten Realismus in Bezug auf identifizierbare Mechanismen, die die kausalen Rollen der Verhaltensdispositionen erfüllen [1]. Allerdings - dies ist unbestritten - gestaltet sich die empirische Einlösung dieses realistischen Anspruchs als schwierig. Einige vorläufige Überlegungen mögen andeuten, dass bereits eine oberflächliche Betrachtung der Zusammenhänge zu einer skeptischen Einschätzung der Erfolgsaussichten führt. Der Weg von empirisch eindeutigen Dispositionen zu konkreten physiologischen Mechanismen, die diesen zugrunde liegen, wird umso steiniger, je stärker deren Manifestationen durch Systemdynamik und Kontext bestimmt wird.

\section{Verhaltensdispositionen manifes- tieren sich situationsabhängig}

Verhaltensdispositionen mit kausalen Mechanismen zu identifizieren, ist per se problematisch. Denn die Bedingungen und Prozesse, die für die Manifestation einer Disposition situativ verantwortlich sind, variieren in Abhängigkeit vom Kontext. Ähnlich dem Zucker, der sich ohne Flüssigkeit nicht auflöst, hängt die Manifestation von Lisas Extrovertiertheit von sozialen
Gelegenheiten ab. Selbst der schüchternste Junge der Gegend, so eine ironische Bemerkung Gilbert Ryles, kann gut und gern als „extrovertiert“ gelten, wenn er unter Bedingungen lebenslanger Einzelhaft beobachtet wird [4]. Somit müssen die zugrunde liegenden Bedingungen bei der kausalen Analyse von Verhaltensdispositionen ,relational“, d. h. in Beziehung mit anderen Bedingungen, verstanden und untersucht werden. Ähnlich der gemessenen Masse eines Objekts, die vom Gravitationsfeld abhängt, erweist sich die Betonung „intrinsischer“, allein aus den organismischen Gegebenheiten erwachsende Eigenschaften als nicht ausreichend. Entscheidend für das Verständnis von Verhaltensdispositionen wären „extrinsische“ Eigenschaften, die aus Wechselwirkungen erwachsen - Wechselwirkungen zwischen physiologischen Mechanismen untereinander und Wechselwirkungen zwischen Organismus und Umwelt.

\section{Verhaltensdispositionen sind viel- dimensionaler und gradueller Natur}

Wie extrovertiert sich Lisa in einer Situation verhält, variiert, weil Lisas Verhalten von verschiedenen Bedingungen abhängt. Zu diesen Bedingungen zählen neben der jeweiligen äußeren Situation (Gottesdienst oder Party) auch interne Bedingungen bzw. Dispositionen (Stimmung, Energielevel etc.). Zudem ist Lisas Extraversion nur eine Dimension ihrer Persönlichkeit - variieren können aber alle Dimensionen.

Würde man Persönlichkeit als Abfolge von messbaren Zuständen analysieren, so bestände die Manifestation von typischen Persönlichkeitsdispositionen in Häufungen bestimmter Merkmalsausprägungen - im Vergleich zu anderen. Es ist deshalb sinnvoll hypothetische Verhaltensdispositionen „kontrastiv“ zu quantifizieren, umso die gesuchten Mechanismen einzugrenzen. Wir fragen, um Lisas Verhaltensdisposition genauer zu bestimmen u. a.: Warum ist Lisa überwiegend extrovertiert und weniger introvertiert (Eigenschaftskontrast)? Warum ist Lisa abends extrovertierter als morgens (zeitlicher Kontrast)? Warum geht Lisa gerne auf Leute zu, während Peter sich z. B. gerne mit seiner Briefmarkensammlung beschäftigt (Situationsbzw. Objekt-Kontrast)? Etc. Je genauer wir „Lisas Extrovertiertheit“ derart eingrenzen, 
umso besser dürften die Aussichten einer mechanistischen Analyse sein. Angesichts der situationsabhängigen Dynamik des Geschehens ist es jedoch schwierig, dies auf ökologisch valide Weise zu tun.

\section{Was ist mit bislang nicht realisierten und sich ändernden Dispositionen?}

Wenn die dispositionsaktivierende Situation oder die dispositionsimplementierenden zerebralen Prozesse selten sind, dann kann es gut sein, dass eine Verhaltensdisposition nie realisiert wird. Selbst Vasen, die nie herabgefallen sind, sind zerbrechlich. Die Möglichkeit von Dispositionen, die sich nicht halbwegs zuverlässig manifestieren, wirft die Frage nach „maskierenden“ oder „kompensierenden“ Bedingungen auf: Warum zerbrach die Ming-Vase nicht, als sie herunterfiel? Weil sie sich in einer professionellen Transport-Box befand, lautet die Erklärung. Warum reagiert dieser „irritierbare“ Typ nicht irritiert? Weil er gelernt hat, seine Irritierbarkeit zu kompensieren. Wir müssen bei der Analyse definierter Verhaltensdispositionen im Blick behalten, dass sich die ursprünglichen Dispositionen verändern und dass kompensierende Fähigkeiten entwickelt werden können. Die Subjekte sind schließlich bemüht, sich anzupassen, dazu zu lernen, sich selbst zu optimieren etc. Ein Blick auf die Veränderbarkeit von Persönlichkeitsdispositionen infolge von Psychotherapie belegt, dass Dispositionsveränderungen bzw. kompensierende Kompetenzerweiterungen nicht zu unterschätzen sind [5].

\section{Wie viele Verhaltensdispositionen gibt es?}

Diese hinterlistige Frage berührt 2 grundlegende Probleme: Erstens, wie individuieren wir Verhaltensdispositionen? Bei näherer Betrachtung wird klar, dass wir bei der Bestimmung von Verhaltensdispositionen weniger von der „organismischen Basis“, als von praktischen Erwägungen ausgehen. Zu diesen praktischen Erwägungen zählen das psychologische Vorverständnis, das Forschungsinteresse, theorieabhängige Fragestellungen, etablierte Experimental- und Messparadigmen, die vorhandenen Ressourcen, die herrschende Forschungs- und Publikationspolitik und Ähnliches mehr. Solange sich Verhaltensdispositionen nicht organismisch eingren- zen lassen, ist die Aufstellung immer neuer Dispositionshypothesen allein durch die Regeln verhaltenswissenschaftlicher Methodik beschränkt.

Zweitens, angesichts vieler verschiedener, womöglich gleichzeitig wirksamer Verhaltensdispositionen stellt sich das systematische Problem der Wechselwirkung zwischen Dispositionen. Ein Blick auf die Ontogenese verdeutlicht die Tragweite des Problems: Aus Zygoten werden Personen, deren Verhaltensdispositionen erfahrungsabhängig ausgeprägt werden. Wir sind Vertreter einer Spezies, die dank ihres sozialen Umfelds lernen, sich und die Umwelt so zu verstehen und zu handhaben, wie man dies tut. Unsere speziestypischen, genetisch angelegten Verhaltensdispositionen werden ohne Frage stark durch interpersonelle, an kulturellen Standards ausgerichtete Lernprozesse ausgeformt. Dies hat u. a. große interindividuelle Unterschiede hinsichtlich Ausprägung und „Legierung“ der vielen verschiedenen Verhaltensdispositionen zur Folge.

\section{Alle Verhaltensdispositionen zusammen ergeben noch keine Person}

Extrovertiert zu sein, bedeutet noch nicht, sich auch extrovertiert zu verhalten. Denn Personen verfolgen Ziele, halten sich an Normen und Priorisieren. Dazu müssen viele Verhaltensbereitschaften adaptiv gemanagt werden. Dies bringt Fragen nach Motivation, Selbstaufmerksamkeit und Selbstkontrolle ins Spiel. Handelt es sich bei diesen Größen ebenfalls um Dispositionen? Oder brauchen Dispositionen, um wirksam zu werden, den „Treibstoff“ der Motivation und die „Kontrolle“ durch Aufmerksamkeit und Steuerung, um situationsangemessen zum Einsatz zu kommen?

Diese und ähnliche Überlegungen zeigen, dass der dispositionalistische Ansatz in einer Zwickmühle steckt: Von der einen Seite droht der Dispositionalismus Opfer eines „anthropomorphen Psychologisierens“ zu werden. Die (anthropomorphe) Art, wie wir uns kulturvermittelt selbst verstehen, sollte nicht einfach auf das physiologische Geschehen projiziert werden. Denn die Art, wie biologische (Teil-)Systeme des vielfach eingebetteten Gehirns sich selbst organisieren, hat nicht viel mit der landläufigen Fakultäten-Psychologie gemein. Begriffe wie Motivation, Aufmerksamkeit, Denken oder Fühlen beschreiben Verhalten; sie bilden keine autochthonen psycho-biologischen Module ab.

Die andere Seite der Zwickmühle könnte man mit dem Prinzip „biologischer Autonomie“ umschreiben [6]. Alle Aktivitäten biologischer Systeme dienen zu allererst dem Selbsterhalt: Zellen müssen die eigene Homöostase gewährleisten, um einen Beitrag zu mehrzelligen Aktivitäten leisten zu können. Die Aktivität der Organe hängt von der Situation des Gesamtorganismus ab. Und auch auf der psychologischen Ebene geht es entscheidend um die Sicherstellung bzw. den Erhalt des Selbst. M. E. steht die reduktionistische Programmatik der dispositionalistischen Analyse gewissermaßen quer zu den Systemaspekten biologischer Selbsterhaltung. Dies allein könnte eine Erklärung für die infrage stehenden Schwierigkeiten sein.

\section{Welche Rolle spielt die Verhaltensintegration?}

Wir erwarten von erwachsenen Personen, sich nicht gehen zu lassen. Die atavistische Manifestation vieler „primitiver“, womöglich „substratnaher“ Verhaltensdispositionen ist unerwünscht. Also sind Erziehung, mühevolle „Selbstdomestizierung“ und adaptive Verhaltenssteuerung gefordert, um sozial akzeptables Verhalten sicher zu stellen. Um dieses zu gewährleisten, müssen Dispositionen „höherer Ordnung“ angenommen werden. Die situative Aktivierung einer „primitiven“ Disposition muss durch die Aktivierung von - sagen wir - „meta-kognitiver Selbstvergegenwärtigung“, von normativ wünschenswerten „Oberzielen“ und dem Einsatz von „Selbststeuerungsfähigkeiten“ eingefangen bzw. integriert werden. Hinreichende Selbststeuerungsfähigkeit - wahrscheinlich ein Bündel von Fähigkeiten bzw. Dispositionen - ist entscheidend für die Einhaltung des Standards einer vernünftigen, psychisch normalen Person.

\section{Was kennzeichnet pathologische Dispositionen, die sich im Verhal- ten durchsetzen?}

Das Standardmodell pathogener Dispositionen besagt: Das Risiko, psychisch zu erkranken, wird durch das Vorliegen bestimmter 
genetischer und neurophysiologischer Bedingungen erhöht. Entsprechende Funktionsstörungen führen zur Manifestation von Symptomen, die in der Form typischer Syndrome kovariieren. Doch die Progression von Risikodispositionen, über pathogene Dispositionen bis zur Manifestation psychopathologischer Symptome ist keine lineare. Ungünstige Entwicklungsbedingungen, unzureichende Fertigkeiten und Ressourcen und „dysfunktionale Selbstbehauptungsstrategien " haben einen erheblichen moderierenden Einfluss auf das Manifestationsrisiko. Es erscheint angesichts der Komplexität und Dynamik des zugrundeliegenden Geschehens schlicht naiv, Symptome als unmittelbaren Ausdruck neuronaler Funktionsstörungen - als „breakdown patterns“ - zu deuten. Vielmehr ist anzunehmen, dass Symptome genauso gut das Ergebnis gescheiterter Kompensationsbemühungen oder Selbstdeutungen sein können. Erkennbar wird dies im Rahmen der psychiatrischen Diagnostik, in der Symptome regelrecht „ausgehandelt“ werden, da der Patient Schwierigkeiten hat, sein Erleben auf den „richtigen“, weil intelligiblen Begriff zu bringen [7].
Interessenkonflikt

Es liegen keine Interessenkonflikte vor.

Korrespondenzadresse

Dr. Markus R. Pawelzik

EOS Klinik für Psychiatrie

Hammer Str. 8

48153 Münster

m.pawelzik@alexianer.de

Literatur

[1] Craver CF, Darden L. In search of mechanisms. Discoveries across the life sciences. Chicago: University Press; 2013

[2] Falkai P, Wittchen H-U. Hrsg. Diagnostisches und Statistisches Manual Psychischer Störungen. Göttingen: Hogrefe; 2015

[3] Uttal WR. The new phrenology. The limits of localizing cognitive processes in the brain. Cambridge, Massachusetts: The MIT Press; 2001

[4] Ryle G. The concept of mind. Chicago: University Press; 2002 (1949)
[5] Roberts BW, Luo J, Briley A et al. A systematic review of personality trait change through intervention. Psychological Bulletin 2017; 143: 117-141

[6] Moreno A, Mossio M. Biological autonomy. A philosophical and theoretica enquiry. Dordrecht: Springer; 2015

[7] Marková IS, Berrios GE. Epistemology of psychiatry. Psychopathology 2012; 45: 220-227

Bibliografie

DOI https://doi.org/10.1055/a-0952-7208 Nervenheilkunde 2019; 38: 965-969 (c) Georg Thieme Verlag KG Stuttgart · New York ISSN 0722-1541 